\title{
EFFECT OF ANTENNA POINTING ERRORS ON SAR IMAGING CONSIDERING THE CHANGE OF THE POINT TARGET LOCATION
}

\author{
X. Zhang ${ }^{1}$, S. J. Liu ${ }^{1, *}$, H. F. Yu², X. H. Tong ${ }^{1}$, G. M. Huang ${ }^{3}$ \\ ${ }^{1}$ College of Surveying and Geo-Informatics, Tongii University, 1239 Siping Road, Yangpu District, Shanghai, China - \\ (1610960, liusjtj, xhtong)@tongji.edu.cn \\ ${ }^{2}$ China Academy of Space Technology, 102 Youyi Road, Haidian District, Beijing, China - castyu2@126.com \\ ${ }^{3}$ Chinese Academy of Surveying and Mapping, 28 Lianhua West Road, Haidian District, Beijing, China - \\ huang.guman@casm.ac.cn
}

Commission III, ICWG III/IVb

KEY WORDS: Synthetic Aperture Radar, Spotlight SAR Mode, Antenna Pointing Errors, Paired Echo, Target Location

\begin{abstract}
:
Towards spaceborne spotlight SAR, the antenna is regulated by the SAR system with specific regularity, so the shaking of the internal mechanism is inevitable. Moreover, external environment also has an effect on the stability of SAR platform. Both of them will cause the jitter of the SAR platform attitude. The platform attitude instability will introduce antenna pointing error on both the azimuth and range directions, and influence the acquisition of SAR original data and ultimate imaging quality. In this paper, the relations between the antenna pointing errors and the three-axis attitude errors are deduced, then the relations between spaceborne spotlight SAR imaging of the point target and antenna pointing errors are analysed based on the paired echo theory, meanwhile, the change of the azimuth antenna gain is considered as the spotlight SAR platform moves ahead. The simulation experiments manifest the effects on spotlight SAR imaging caused by antenna pointing errors are related to the target location, that is, the pointing errors of the antenna beam will severely influence the area far away from the scene centre of azimuth direction in the illuminated scene.
\end{abstract}

\section{INTRODUCTION}

The stable control towards the attitude of SAR platform is a crucial factor concerning the imaging quality. As the SAR platform moving, both external environment and internal interference cause the antenna pointing errors. Meanwhile, Spotlight SAR is a commonly used SAR work mode. Comparing to the stripmap SAR, spotlight SAR achieves much higher resolution by controlling the antenna to point a settled scene. So antenna pointing errors get more severe due to the shaking of the internal mechanism during the antenna pointing adjustment process. The effect on image qualities caused by the Satellite attitude instability has been analysed based on paired echo theory (Huang et al., 2000; Chen et al., 2001), both of those analysis are applying to the stripmap mode with sidelooking incident angle and considering the single jitter frequency. Zhu et al. (2006) have analysed the effect on equivalent squint mode SAR caused by multi frequency attitude jitter. Wang et al. (2007) and Qi et al. (2013) have discussed the effect of attitude jitter on spotlight mode SAR, but both do not consider the change of the antenna beam pointing direction over time. Giudici et al. (2008) have analysed the effects of static and dynamic errors of antenna beam pointing and predicted their impact of the most relevant SAR image quality parameter, which do not combine the paired echo theory.

So this paper analyses the effect of the antenna pointing errors caused by platform jitter on above mentioned spotlight SAR mode based on paired echo theory. The paper includes five parts. The background is presented in Section 1. The models of antenna beam pointing jitter towards spotlight SAR mode are built in Section 2.1. The effects of antenna beam pointing jitter are deduced based paired echo theory in Section 2.2. Then the simulation experiments of effects on SAR imaging caused by the azimuth and the range direction antenna pointing jitter are introduced in Section 3. Finally, conclusions are presented in Section 4.

\section{METHODOLOGY}

\subsection{Mode of Antenna Jitter towards different SAR modes}

The planar imaging geometry of spotlight SAR is shown in Figure1 (Qi et al., 2013). The SAR Sensor travels along the azimuth direction with the velocity of $V$. The synthetic aperture centre is at point $O$, at which the antenna beam points in a vertical direction. In the spotlight $\mathrm{SAR}$, the beam point centre is fixed at the scene centre $O^{\prime} . P$ is a point target at $\left(x_{0}, R_{0}\right)$, where $R_{0}$ is the vertical range between the radar platform and ground target. With the movement of the SAR sensors, especially when the platform moves to the $X$ in time $t, \theta_{k}$ is defined as the included angle between the beam centre and target $P$.

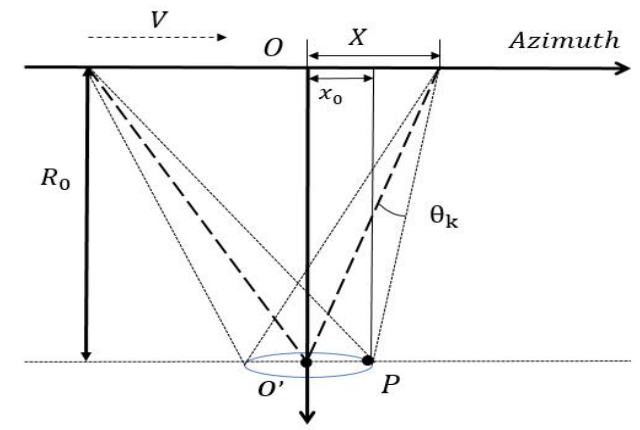

Figure 1. Illuminated geometry of spotlight SAR

The mathematical expressions of $\theta_{k}$ are as formula (1), considering the adjustment of antenna centre direction as a 
continuous process, which do not influence the regularity of results.

$$
\theta_{k}=\arctan \left(\frac{V t}{R_{0}}\right)+\arctan \left(\frac{\mathrm{x}_{0}-V t}{R_{0}}\right)
$$

On the other hand, the relationship between three-axis attitude error and the antenna beam pointing errors is deduced based on the transformation of the platform ontology coordinate system to the ground solid coordinate system, the mathematical expressions are as follow:

$$
\begin{gathered}
\Delta \theta_{a}=\left(\cos \theta_{L} \delta_{p}-\sin \theta_{L} \delta_{y}\right) \\
\Delta \theta_{r}=\frac{\left(\delta_{y} \sin \theta-\cos \theta \cos \theta_{L} \delta_{r}+\cos \theta \sin \theta_{L}\right) \div \cos \theta-\sin \theta_{L}}{\cos \theta_{L}}
\end{gathered}
$$

where $\delta_{y}$ is the angle error in yaw direction, $\delta_{p}$ is the angle error in pitching direction, $\delta_{r}$ is the angle error in rolling direction, $\theta$ is the squint angle, $\Delta \theta_{\mathrm{r}}$ is the angle error in range, and $\Delta \theta_{a}$ is the angle error in azimuth. It is obvious that the antenna pointing angle errors in azimuth and range direction are not linear relation with the three-axis attitude jitter errors, so in the simulation experiment, the final antenna pointing angle errors of azimuth and range direction are given.

\subsection{Effect of Antenna Pointing Jitter on Image Quality}

According to the paired echo theory (Huang et al., 2000), the platform attitude jitter will cause the pointing errors of antenna beam, then bring the paired echo in azimuth, exacerbate the image blur, lower resolution and produce false targets. After the image processing, the azimuth echo can be simplified to the signal mode:

$$
s(t)=w_{a}(t) w_{r}(t) e^{-j \pi f_{r} t^{2}}
$$

where $w_{a}(t)$ stands the antenna radiation pattern in range direction, while $w_{r}(t)$ in azimuth direction.

After the phase compensation and antenna weighting processing in the imaging process, the final target signal is (Huang et al., 2000):

$$
S_{m}(t)=e^{-j \pi f_{r} t^{\wedge 2}} \int_{-\infty}^{\infty} w_{a}(t) w_{r}(t) e^{-j 2 \pi f_{r} \cdot \tau} d \tau
$$

where $f_{r}$ is the Doppler rate.

When existing the attitude error, the output point response is $s_{0 m}(t)$ after the imaging process (Huang et al., 2000).

$$
s_{f a}(t)=s_{m}(t)-s_{w a}(t)
$$

where $s_{w a}(t)$ is the pair-echoes, $s_{m}(t)$ is the main beam.

2.2.1 Azimuth antenna pointing jitter Suppose the direction of the antenna beam wobbles as follows:

$$
\Delta \theta_{a}(t)=\theta_{a m} \sin \left(\omega_{0} t+\varphi_{0}\right)
$$

$\theta_{a m}$ is the amplitude of the azimuth antenna jitter caused by yaw and pitch angle, $\omega_{0}$ is the angular frequency of the jitter. So the azimuth antenna gain is given by (Huang et al., 2000):

$$
w_{a}(t)=w_{a}\left[\theta_{a 0}+\theta_{a m} \sin \left(\omega_{0} t+\varphi_{0}\right)\right]
$$

where $\theta_{a 0}$ is the Off-axis Angle of the target in azimuth direction, it is equal to $\theta_{k}$ when considering the spotlight SAR mode. For any moment, because the antenna jitter angle is far smaller than the original point Angle, using a Taylor series expansion on $\theta_{k}$ and ignoring the high term, then $w_{a}(t)$ can be represented as the $w_{a 1}(t)$ (Huang et al., 2000):

$$
w_{a 1}(t) \cong w_{a}\left(\theta_{k}\right)+w_{a}{ }^{\prime}\left(\theta_{k}\right) \theta_{a m} \sin \left(\omega_{0} t+\theta_{0}\right)
$$

Set $w_{r}\left(\theta_{r 0}\right)$ as the antenna gain of distance direction, the final target output signal can be expressed as (Huang et al., 2000):

$$
\begin{aligned}
s_{f a}(t)= & w_{r}\left(\theta_{r 0}\right) e^{j \pi f_{r} t^{\wedge} 2} \int_{-\infty}^{\infty} w_{a 1}(\tau) e^{-j 2 \pi f_{r} t . \tau} d \tau \\
= & w_{r}\left(\theta_{r 0}\right) e^{j \pi f_{r} \wedge 2} \int_{-\infty}^{\infty}\left[w_{a}\left(\theta_{k}\right)\right. \\
& \left.+w_{a}{ }^{\prime}\left(\theta_{k}\right) \theta_{a m} \sin \left(\omega_{0} t+\theta_{0}\right)\right] e^{-j 2 \pi f_{r} t . \tau} d \tau
\end{aligned}
$$

The main echo is:

$$
S_{m}(t)=w_{r}\left(\theta_{r 0}\right) e^{j \pi f_{r} \wedge 2} \int_{-\infty}^{\infty} w_{a}\left(\theta_{k}\right) e^{-j 2 \pi f_{r} t . \tau} d \tau
$$

The paired echo is:

$$
\left.S_{w a}(t)=w_{r}\left(\theta_{r 0}\right) e^{j \pi f_{r} \wedge^{\wedge} 2} \int_{-\infty}^{\infty} w_{a}\left(\theta_{k}\right) \theta_{a m} \sin \left(\omega_{0} \tau+\theta_{0}\right)\right) e^{-j 2 \pi f_{r} t . \tau} d \tau
$$

Chen et al. (2001) ignore the change of the antenna gain over time, accordingly get the conclusion that the paired echoes appears at $\pm f_{0} / f_{r}$. Actually, the antenna gain towards stationary target is changing as the platform goes ahead. Especially, the antenna pointing is controlled by the SAR system in spotlight mode, the azimuth antenna gain changes more complicated as the form of $w_{a}\left(\theta_{k}\right)$ over time. So the relation between the azimuth direction antenna pointing jitter frequency and the paired echo peak locations is no analytic regularity. Particularly, when the target locates at scene centre, the derived function of antenna gain is 0 in spotlight SAR, for the antenna beam points to scene centre all the time, accordingly, the paired echo will be constant 0 .

2.2.2 Range antenna pointing jitter

The antenna gain in the distance direction caused by the roll has the same influence process as the azimuth direction, antenna gain in range direction is:

$$
w_{r}(t)=w_{r}\left[\theta_{r 0}+\theta_{r m} \sin \left(\omega_{0} t+\theta_{0}\right)\right]
$$

where $\theta_{r 0}$ is the Off-axis angle of the target in range direction, in spotlight SAR mode, the range direction off-axis angle towards certain target will not change as the SAR platform moving ahead, which is different from the azimuth direction off-axis angle. So in the parsing expression, range direction 
antenna jitter will cause different regularity. Similarly, the final target output signal can be expressed as (Chen et al., 2001)

$$
\begin{aligned}
s_{f r}(t)= & e^{j \pi f_{r} \iota^{\wedge} 2} \int_{-\infty}^{\infty} w_{a}(\tau)\left[w_{r}\left(\theta_{L}\right)\right. \\
& \left.+w_{r}\left(\theta_{L}\right) \theta_{r m} \sin \left(\omega_{0} t+\theta_{0}\right)\right] e^{-j 2 \pi f_{r} t . \tau} d \tau
\end{aligned}
$$

For the antenna gain of the range direction is no relation with the azimuth time, the paired echo can be expressed as (Wang et al., 2007):

$$
\begin{aligned}
S_{w r}(t)= & \frac{w_{r}\left(\theta_{L}\right) \theta_{r m}}{2 j} e^{j \pi f_{r} t^{2}}\left[e^{-j \theta_{0}} S_{0}\left(t+\frac{f_{0}}{f_{r}}\right)\right. \\
& \left.-e^{j \theta_{0}} s_{0}\left(t-\frac{f_{0}}{f_{r}}\right)\right]
\end{aligned}
$$

where $S_{0}(t)=\int_{-\infty}^{\infty} w_{a}(\tau) e^{-j 2 \pi f_{r} t . \tau} d \tau$

Chen et al. (2001) and Wang et al. (2007) also consider the paired echoes appears at $\pm f_{0} / f_{r}$. Similarly, the azimuth antenna gain changes over time, $w_{a}(\tau)$ is not a constant. So the relation between the range direction antenna pointing jitter frequency and the paired echo peak locations is also no analytic regularity. According to the above analysis, the greater the amplitude of the antenna pointing jitter, the higher the peak of the paired echo when other simulation parameters are equal, and the regularity has already been verified. So the simulation experiment mainly considers the influence towards the amplitude of the paired echo caused by the target location. Since the regularities of the paired echoes caused by the azimuth and range pointing jitter antenna are similar, only the azimuth results have been given.

\section{SIMULATION RESULTS}

According to the above analysis, the effects of attitude jitter on image quality are simulated in this section, and the effect caused by azimuth direction jitter is different from the one of range direction. So the simulation results are divided into two parts, and the simulation parameters are shown as Table 1.

\begin{tabular}{|l|c|}
\hline Parameter (unit) & Value \\
\hline Orbit altitude $(\mathrm{km})$ & 700 \\
Wave length $(\mathrm{m})$ & 0.03 \\
Velocity $(\mathrm{km} / \mathrm{s})$ & 7.5 \\
Antenna aperture $(\mathrm{m})$ & 10 \\
Pulse repetition frequency $(\mathrm{Hz})$ & 5000 \\
\hline
\end{tabular}

Table 1. Simulation parameters

From the equation (2) and (3), the angle of antenna direction is coupling with the attitude jitter angle. So in the simulation, the final antenna jitter angles of azimuth and range direction were given. Besides, the frequency of the jitter behaves no analytical law towards the spotlight SAR by above analysis, refer to Chen et al., (2001), give the antenna jitter parameters that antenna jitter amplitude of the azimuth direction is $0.05 \mathrm{deg}$, the frequency is $4 \mathrm{~Hz}$ without loss of generality, $\left(0, Y_{c}\right)$ is the scene centre position of the target scene, and the simulation results are shown as follow. For each Figure showing the echo, the above part represents the dimensionless amplitude of the echo, considering the backscatter coefficient is 1 , and the nether one denotes the normalized power with the unit DB.

\subsection{Azimuth direction antenna beam pointing jitter}

When the target locates in the scene centre $\left(0, Y_{c}\right)$, the simulation results are displayed in Figure 2.
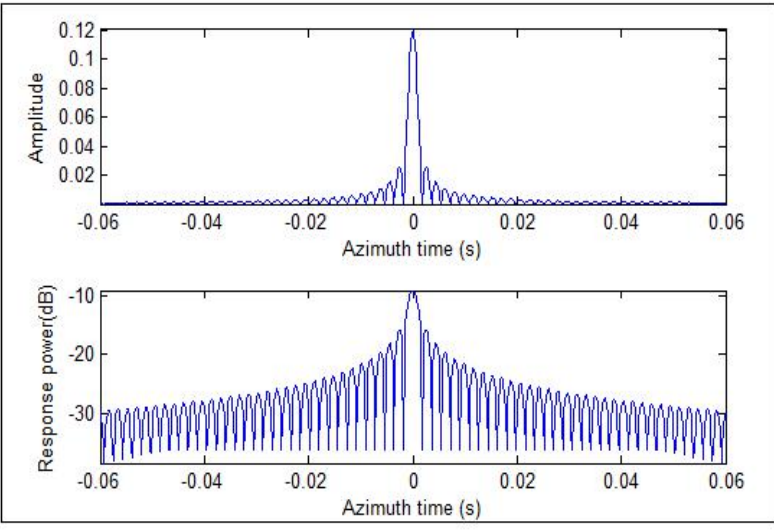

(a) Original echo
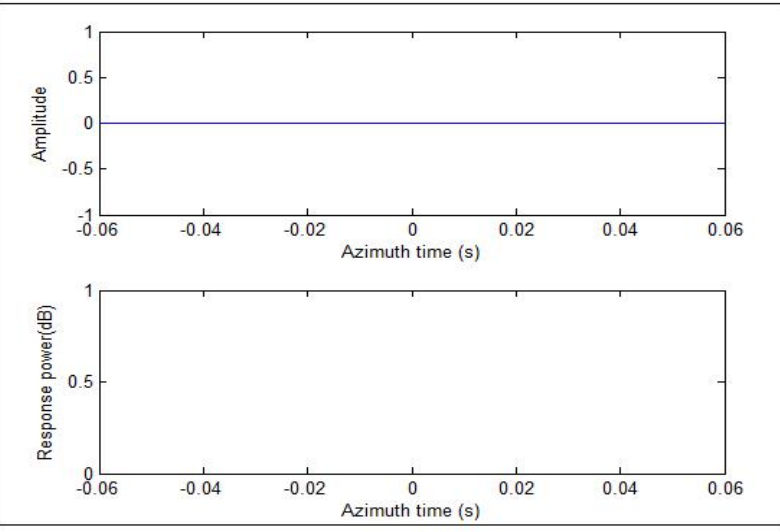

(b) Echo cause by antenna pointing jitter
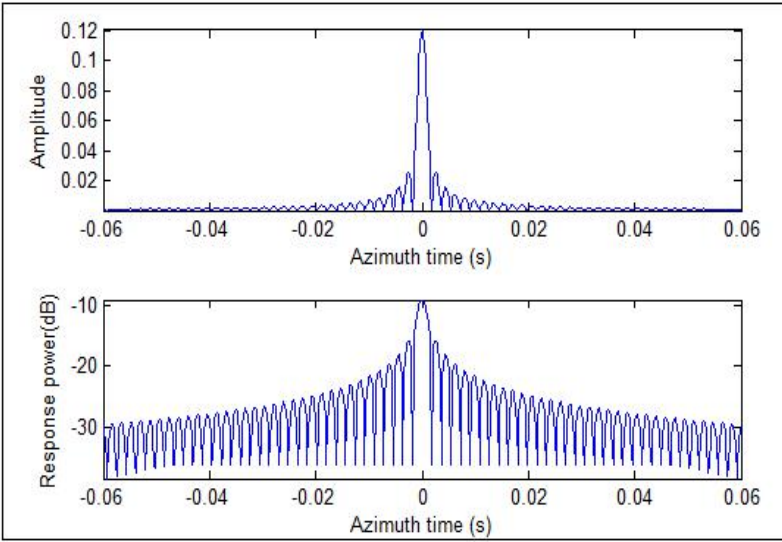

(c) Final echo

Figure 2. Simulation results of the echoes before and after azimuth direction pointing jitter towards target $\left(0, Y_{c}\right)$ 


\begin{tabular}{|c|c|c|}
\hline Statistical index & $\begin{array}{c}\text { Original ideal } \\
\text { echo }\end{array}$ & $\begin{array}{c}\text { Echo after antenna } \\
\text { pointing jitter }\end{array}$ \\
\hline PSLR & $-13.274 \mathrm{~dB}$ & $-13.274 \mathrm{~dB}$ \\
ISLR & $-10.6945 \mathrm{~dB}$ & $-10.6945 \mathrm{~dB}$ \\
\hline
\end{tabular}

Table 2. Compare of reference index

According to the simulation results, the paired echo will not appear, that is, azimuth antenna pointing jitter almost cause no influence to the original echoes. This is because the derivative of the antenna gain is constant zero, for the antenna always points to the target in the scene centre as the SAR platform moving ahead. When the target locate in $\left(1000, Y_{c}\right)$, the simulation results are shown in Figure 3.

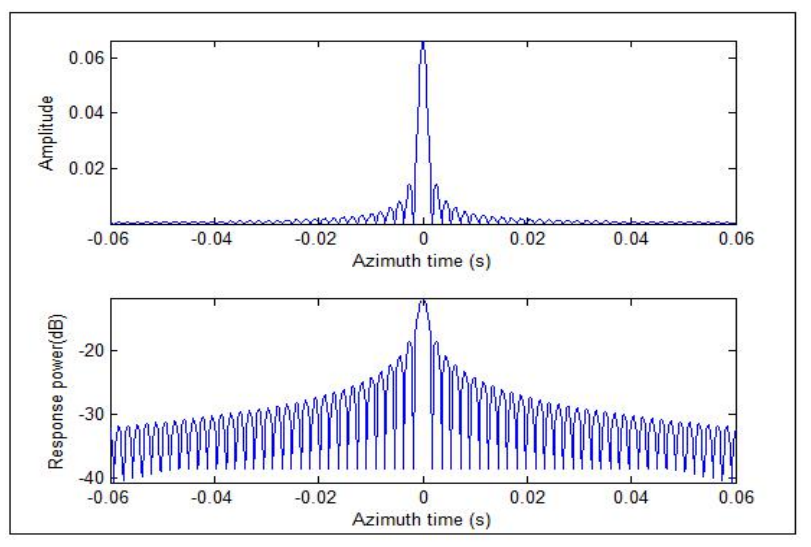

(a) Original echo

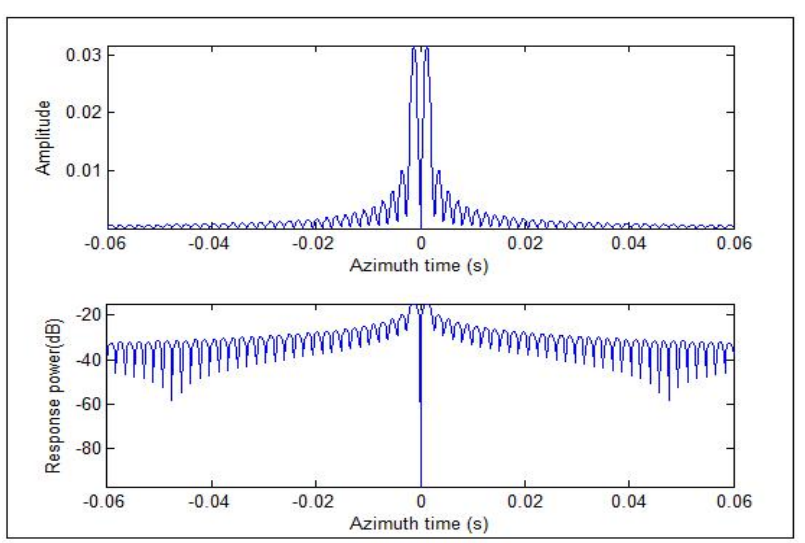

(b) Echo cause by antenna pointing jitter

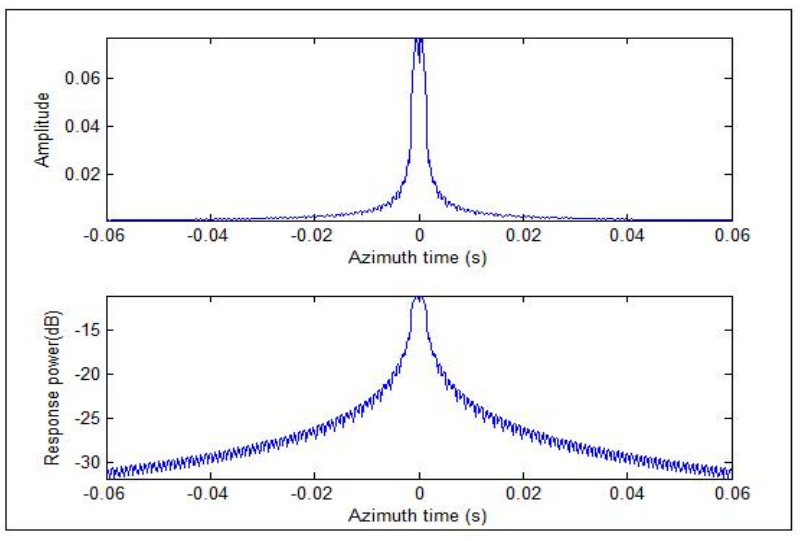

(c) Final echo

Figure 3. Simulation results of the echoes before and after azimuth direction pointing jitter towards target $\left(1000, Y_{c}\right)$

\begin{tabular}{|c|c|c|}
\hline Statistical index & $\begin{array}{c}\text { Original ideal } \\
\text { echo }\end{array}$ & $\begin{array}{c}\text { Echo after antenna } \\
\text { pointing jitter }\end{array}$ \\
\hline PSLR & $-13.274 \mathrm{~dB}$ & $1.3013 \mathrm{~dB}$ \\
ISLR & $-9.1252 \mathrm{~dB}$ & $-6.3 \mathrm{~dB}$ \\
\hline
\end{tabular}

Table 3. Compare of reference index

From the simulation results, the paired echo caused by the antenna pointing jitter is symmetrical. When the target locates away from the scene centre in azimuth, the peak of the paired echo caused by the pointing jitter in the azimuth direction gets higher, PSLR and ILSR raise obviously, which indicate the reduction of the imaging quality.

\subsection{The influence of the azimuth location to azimuth direction antenna pointing jitter simulation}

For azimuth direction antenna pointing jitter, the coordinate change in range direction only influences the range direction antenna gain amplitude of the echo. So consider that the azimuth direction coordinate of the target location is from $1000 \mathrm{~m}$ to $1000 \mathrm{~m}$, and the step is $20 \mathrm{~m}$. The results are as Figure 4.

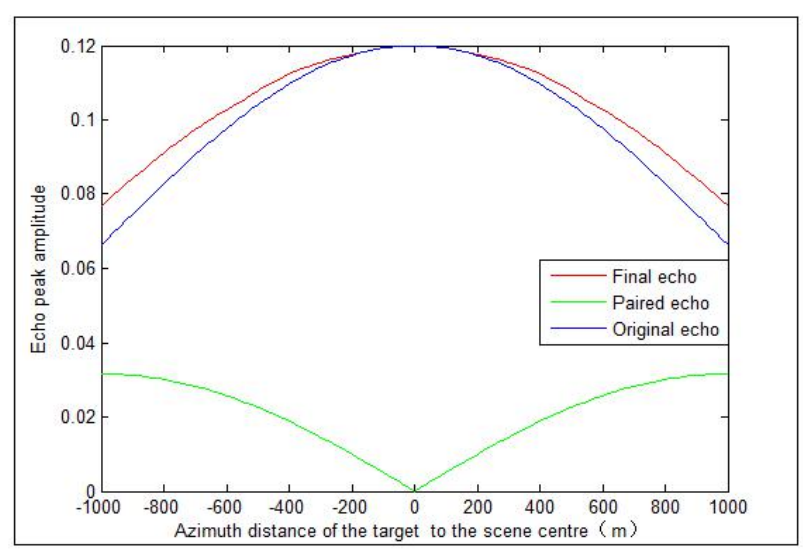

Figure 4. The influence of target azimuth direction position to the echo peak amplitude

When the target location of azimuth direction gets away from the centre $\left(0, Y_{c}\right)$, the peak amplitude of the original echo and 
final echo reduce, paired echo increases in a symmetrical tendency correspondingly.

\subsection{The influence of the range location to azimuth direction antenna pointing jitter simulation}

Towards range direction beam jitter, make the azimuth coordinate $500 \mathrm{~m}$, consider that the azimuth direction coordinate of the target location is from $Y_{c}-1000 \mathrm{~m}$ to $Y_{c}+1000 \mathrm{~m}$, and the step is $20 \mathrm{~m}$. The results are as follow.

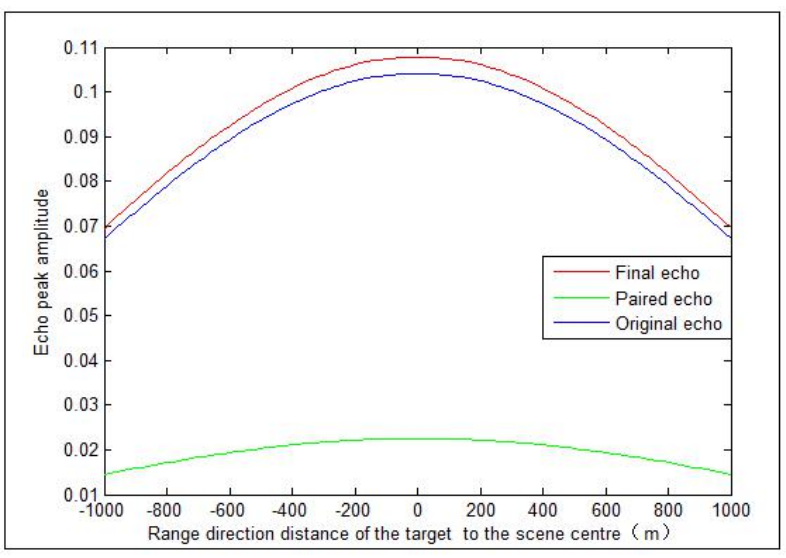

Figure 5. The influence of target range direction position to the echo peak amplitude

Opposite to the target location change in azimuth direction, when the target location of range direction gets away from the centre $\left(0, Y_{c}\right)$, though the peak amplitude of the original echo and final echo reduce, paired echo also reduce symmetrically, this is reason that the term of the range direction antenna gain lowers as the target leaves away the centre $\left(0, Y_{c}\right)$ in range direction. Besides, when the target locates at the centre of the range direction $Y_{c}$, for the azimuth direction coordinate of the target is $500 \mathrm{~m}$, and the derived function of the azimuth direction antenna gain is not a constant 0 , thus the paired echo is not zero in $\left(500, Y_{c}\right)$, shown as the original point of the abscissa axis in Figure 5 .

\section{CONCLUSIONS}

This paper analyses the effect of antenna pointing errors on the spotlight SAR imaging based the paired echo theory. It is approved that the satellite attitude jitter will bring the paired echo, the amplitude of which is decided by the target location. In spotlight SAR mode, the antenna pointing direction is regulated as the platform moving ahead, so both the analytical expression of the paired echo and final simulation results show the otherness in contrast with early researches towards strip map SAR and spotlight SAR. The pointing errors of the antenna beam will influence severely the area far away from the scene centre of azimuth direction in the illuminated scene, but the paired echo lowers gradually when away from the centre of range direction. Therefore, in the design of spotlight SAR system, considering and seeking a way to compensate the negative effect of the attitude jitter is necessary.

In future research, the influence on face target caused by the antenna pointing jitter will be considered.

\section{REFERENCES}

Huang, Y., Li, C. S., Chen, J., 2000. Effects of Stability of Antenna Pointing on Space borne SAR Imaging Quality. Journal of Beijing University of Aeronautics \& Astronautics, 26(3):282-285.

Chen, J., Zhou, Y. Q. and Li, C. S., 2001. On the Relationship between Satellite Attitude Stability and Space-borne SAR Image Qualities, ACTA ELECTRONICA, pp. 1785-1789.

Zhu, L., Qiu, G. F., Pan, J., 2006. Analysis of Antenna Bore sight Pointing Instability Effect on SAR Image Quality. Modern Radar, 28(1):61-64.

Wang, P. B., Zhou, Y. Q., Chen, J., 2007. Effect of Satellite Attitude Stability on Spotlight SAR Imaging. Modern Radar.

Giudici, D., D'Aria, D., Guarnieri, A. M., et al, 2008. Analysis of antenna pointing errors on SAR image quality. Radar Conference. RADAR '08. IEEE, 1-6.

Qi, Y. L., Zhang, Q. J., Guo, J. J., 2013. Effect of satellite attitude errors on spotlight SAR image. Radar Conference 2013, IET International. IET, 1-5. 